\section{Difference-Differential Equations}

THE general linear homogeneous differencedifferential equation with constant coefficients is

$$
\Lambda(y) \equiv \sum_{\mu \nu} a_{\mu \nu} y^{(v)}\left(t+b_{\mu}\right)=0,
$$

where $0 \leqslant \mu \leqslant m, 0 \leqslant \nu \leqslant n, y^{(v)}(t)$ is the $v$-th derivative of the unknown function $y(t)$ and $0=b_{0}<b_{1}<. . .<b_{m}$. Particular examples of this equation have appeared in radio$\log y^{1,2}$, economics ${ }^{3,4}$ and the theory of control mechanisms ${ }^{5,6}$. The most useful 'boundary conditions' are also the most convenient from the theoretical point of view; we suppose assigned the values of $y(t)$ in an initial interval $0 \leqslant t<b_{m}$. In terms of these given values, we define a function

$$
\begin{gathered}
\varphi(s)=\sum_{\mu} \sum_{\nu} a_{\mu \nu} \exp \left(b_{\mu} s\right) \times \\
\left\{\int_{0}^{b_{\mu}} y^{(v)}(u) e^{-s u d} d u+{ }_{\lambda=0}^{\nu} s^{v-1-\lambda} y^{(\lambda)}(0)\right\}
\end{gathered}
$$

(In particular cases this usually reduces to something fairly simple.) It is obvious that $y=\exp s t$ is a solution of (1) for any $s$ satisfying

$$
\tau(s) \equiv \sum_{\mu \nu} \sum_{\nu} a_{\mu \nu} s^{\nu} \exp \left(b_{\mu} s\right)=0 .
$$

The zeros of $\tau(s)$ are infinite in number; but their asymptotic behaviour is readily calculable? Under suitable conditions, the solution of (1) is

$$
y(t)=\sum_{\delta} \frac{\varphi(s) \exp s t}{\tau^{1}(s)},
$$

where $s$ runs through all the zeros of $\tau(s)$. I here assume that $\tau(s)$ has no double zero; if it has, a slight modification must be made in the corresponding term. The series in (2) is convergent and its sum is $y(t)$ (i) for all $t$, if $a_{m n} \neq 0$ and $a_{0 n} \neq 0$, and (ii) for all $t>b_{m}$, if $a_{m n} \neq 0$. (2) was first given by Hilb8, but under conditions which would exclude most of the applications. A detailed proof of its validity under the conditions stated will be published shortly ${ }^{\circ}$.

The most interesting question about $y(t)$ is how it behaves when $t$ is large and, in particular, whether $y(t)$ becomes small, periodic or large. This requires a study of the zeros of $\tau(s)$. In any particular case the behaviour of the large zeros can be determined from Langer's theory? ${ }^{7}$. If these zeros all have their real parts negative, so that the corresponding terms in $y(t)$ are damped, attention must be directed to a finite number of non-large zeros. This problem for the particular cases in which

$$
\tau(s) \equiv s(s+\alpha) e^{8}+\beta_{1}+\beta_{2} s+\beta_{s} s^{2}
$$

and

$$
\tau(s) \equiv s^{2}\left(s+\alpha_{1}\right) e^{s}+\left(\beta_{1}+\beta_{2} s\right)\left(\alpha_{1}+\alpha_{2} s\right)
$$

has been discussed by Hartree and others ${ }^{5,8}$. Another special case, into which the equations occurring in radiology ${ }^{1}$ and in economics ${ }^{3,4}$ may be trivially transformed, is the equation

$$
y^{\prime}(t+1)+\alpha y(t)=0 \quad(\alpha>0) .
$$

For this, $\tau(s) \equiv s e^{s}+\alpha$. If $\alpha<\frac{1}{2} \pi$, all zeros of $\tau(s)$ have their real parts negative and $y(t) \rightarrow 0$ as $t \rightarrow+\infty$, whatever the initial conditions. If $\alpha>\frac{1}{2} \pi$, at least two zeros of $\tau(s)$ have their real parts positive and $y(t)$ oscillates with increasing amplitude as $t \rightarrow+\infty$, except under very special initial conditions. For $\alpha=\frac{1}{2} \pi$, there is a periodic solution $y=A \sin \left(\frac{1}{2} \pi t+B\right)$ with arbitrary $A$ and $B$, and the general solution approaches this as $t$ becomes large. There is a periodic solution for $\alpha=2 k \pi+\frac{1}{2} \pi$, where $k$ is any whole number, but the general solution oscillates infinitely unless $k=0$.

The non-homogeneous equation

$$
\Lambda(y)=v(t)
$$

where $v(t)$ is a known function, can also be solved for those $v(t)$ which have a readily calculable Laplace transform. Of course, if any particular solution of (3) can be found, the problem of finding the general solution, or the solution satisfying 'boundary conditions' of our type, is reduced to the same problem for (1), just as in the theory of differential equations.

More substantial and more interesting problems are presented by linear difference - differential equations with variable coefficients ${ }^{10}$ and by non-linear equations ${ }^{11}$. The only published application of such a non-linear equation known to me occurs in Lord Cherwell's investigations by probability methods into the distribution of prime numbers ${ }^{12}$.

Department of Mathematics,

E. M. WRIGHT

University of Aberdeen. April 26.

${ }^{1}$ Sievert, R. M., Acta Radiologica, 22, 237 (1941).

2 van der Werff, J. T., Acta Radiologica, 23, 603 (1942).

${ }^{3}$ Frisch, R., and Holme, H., Econometrica, 3, 225 (1935).

4 James, R. W., and Belz, M. H., Econometrica, 4, 157 (1936).

${ }^{5}$ Callender, A., Hartree, D. R., and Porter, A., Phil. Trans. Roy. Soc., A, 28 ; , 415 (1936). - Hartree, D. R., Porter, A., Callender, A., and Stevenson, A. B.,
Proc. Roy. Soc., A, 161, 460 (1937).

'Langer, R. E., Trans. Amer. Math. Soc., 31, 837 (1929).

${ }^{8}$ Hilb, E., Math. Ann., 78, 137 (1918).

-Wright, E. M., Proc. Roy. Soc., Edin. (in the press).

10 Wright, E. M., Amer. J. Math., 70, 221 (1948).

${ }^{11}$ Wright, E. M., Quart. J. Math., 68, 245 (1946).

12 Lord Cherwell, Nature, 150, 121 (1942).

\section{X-Ray Diffraction Effect Shown by some Specimens of Chrysotile}

IN a study of the 'tailed' spots occurring on X-ray fibre photographs of chrysotile a camera with a slit approximately $0.7 \mathrm{~cm}$. high was used, so that nearly $1 \mathrm{~cm}$. of the fibre was irradiated. The purpose was to obtain lines instead of spots in the X-ray photo. graph, and thus facilitate photometry. The lines obtained, however, were beaded, the effect being particularly evident with specimens from Barberton (Figs. $A$ and $B$ ). A specimen from Thetford showed only a slight variation in intensity along the lines (Fig. $C$ ), and a Russian specimen showed no visible variation. The effect can b甲 imitated to some extent by pinching at regular intervals a specimen not showing it (Fig. D).

The beadiness seems to be due to a fairly regular variation of the scattering power along the fibres, possibly due to variation in external conditions during their growth. It is not due to multiple foci in the $\mathrm{X}$-ray tube or irregularities in the slit system, since the beads move up and down with the specimen when its position is changed; they are not shown by all specimens, and are independent of the tube and camera used. It is unlikely that they are caused by non-uniform mechanical deformation of the fibres in detaching them from the lump, since they were shown by a specimen $0.10 \mathrm{~cm} . \times 0.14 \mathrm{~cm}$. in cross-section, which was quite rigid and could not bend to any extent during detachment. 